\title{
NONLINEAR AND DIRECTION CONNECTIONS
}

\author{
JAAK VILMS ${ }^{1}$
}

\begin{abstract}
Nonlinear connections and direction connections are two types of connections arising in Finsler geometry. In his work on generalized sprays, P. Dazord showed that there is a relationship between these two types (nonlinear connections were called sections by him). This relationship has also been used by J. Grifone in a work on prolongation of direction connections. In this paper we examine this relationship in a general setting. In particular, we show that E. Cartan's condition " $D$ " is necessary and sufficient for a direction connection to define a nonlinear one. Also, we prove a nonuniqueness result for direction connections associated to a given nonlinear one.
\end{abstract}

1. Connections on vector-bundles. We first recall our definition of a connection on a vector bundle [5], [6]. For a smooth $\left(C^{\infty}\right)$ vector bundle $p: E \rightarrow M$, set $E_{0}=E-0$ and $p_{0}=p \mid E_{0}$. The bundle $p^{-1} E$ over $E$ is canonically isomorphic to the bundle $V E$ of vertical vectors in the tangent bundle $T E$ of $E$. Hence we have the exact sequence

$$
0 \rightarrow p^{-1} E \stackrel{J}{\rightarrow} T E \stackrel{p^{\prime}}{\rightarrow} p^{-1} T M \rightarrow 0
$$

of vector bundles over $E$, where $J$ corresponds to the inclusion map $V E \subset T E$ and $p^{\prime}$ is essentially the tangent map $p_{*}: T E \rightarrow T M$.

A smooth nonlinear connection on the vector bundle $p: E \rightarrow M$ is a smooth splitting of (1) over $E_{0}$. Since $T E \mid E_{0}=T E_{0}$ and $p^{-1} E \mid E_{0}$ $=p_{0}^{-1} E$, such a splitting is given by a smooth linear map $V: T E_{0}$ $\rightarrow p_{0}^{-1} E$ (i.e. continuous linear on the fibres), satisfying the equation $V J=$ id. The splitting can be conveniently described by its connection map $D: T E_{0} \rightarrow E$ defined as $D=r \circ V$, where $r: p^{-1} E \rightarrow E$ is the canonical surjection over p. $D$ is continuous linear on the fibres and is smooth.

The connection on $p: E \rightarrow M$ is homogeneous (resp. linear) if the map $D$ is homogeneous of degree 1 (resp. linear) on the $p_{*}$ fibres of $T E$. For a linear connection, the splitting of (1) automatically extends to all of $E$; in fact, a linear connection can be defined as a splitting of (1) which is smooth over all of $E$.

Received by the editors June 23, 1970.

AMS 1969 subject classifications. Primary 5385, 5350.

Key words and phrases. Finsler geometry, nonlinear connection, direction connection, E. Cartan's condition "D", spray.

1 Supported by the NSF. 
Let us now introduce local coordinates. Suppose locally $T M$ and $E$ are isomorphic to product bundles $U \times B$ and $U \times E$, respectively, where $B$ and $E$ are real Banach spaces (the reader should substitute $B=R^{n}, E=R^{m}$ if he is not familiar with calculus in Banach spaces) and $U \subset B$ is an open set. Then $T E_{0}$ is locally isomorphic to the product bundle $\left(U \times E_{0}\right) \times B \times E$ (where $\left.E_{0}=E-0\right)$, and fiberwise for each $(x, a) \in U \times E, D$ is defined by a map $D_{(x, a)}: B \times E \rightarrow E$ of the form

$$
D_{(x, a)}(\lambda, b)=b+\omega(x, a) \lambda .
$$

Here $\omega: U \times E_{0} \rightarrow L(B, E)$ is a smooth map ( $L$ denotes a space of continuous linear maps). $\omega$ is called a local component of the connection.

The connection is homogeneous (resp. linear) iff each $\omega$ is homogeneous of degree 1 (resp. continuous linear) in the second variable, $a$. (Hence for a linear connection we can define $\omega(x, 0)=0$ and obtain a smooth map on $U \times E$.)

2. Linear connections on $p_{0}^{-1} E \rightarrow E_{0}$. Next we consider a linear connection on the vector bundle $p_{0}^{-1} E \rightarrow E_{0}$ and define its torsion.

Since $p_{0}^{-1} E$ is locally isomorphic to $\left(U \times E_{0}\right) \times E$, and $T\left(p_{0}^{-1} E\right)$ to $\left(\left(U \times E_{0}\right) \times E\right) \times(B \times E) \times E$, the connection map $D: T\left(p_{0}^{-1} E\right) \rightarrow p_{0}^{-1} E$ of such a connection is, fiberwise for each $((x, a), b) \in\left(U \times E_{0}\right) \times E$, given by a map $\mathscr{D}_{((x, a), b)}:(B \times E) \times E \rightarrow E$ of the form

$$
\mathscr{D}_{((x, a), b)}((\lambda, c), d)=d+\Omega((x, a), b)(\lambda, c) .
$$

$\Omega$ is continuous linear in $(\lambda, c)$ and, the connection being linear, also in $b$. Hence $D$ must actually be locally of the form

$$
D_{((x, a), b)}((\lambda, c), d)=d+\Gamma_{(x, a)}(b, \lambda)+C_{(x, a)}(b, c),
$$

where $\Gamma, C$ are smooth maps of $U \times E_{0}$ into $L^{2}(E, B ; E)$ and $L^{2}(E, E ; E)$, respectively ( $L^{2}$ denotes a space of continuous bilinear maps).

Although the two parts $\Gamma$ and $C$ of the local component of $D$ do not, of course, transform together as a tensor under a change of coordinates, it happens that the $C$ part does define a tensor, which corresponds to the Finsler torsion tensor of $[1]$. (Since $p^{-1} E \approx V E$, one can transcribe the coordinate change equation of $[6, p .1127]$ to the present notation and set $\mu=0$ to see this fact.) Thus there is a smooth section $J$ of the bundle of bilinear maps from $p_{0}^{-1} E$ to $p_{0}^{-1} E$, which is locally given by maps $\Im_{(x, a)} \in L^{2}(E, E ; E)$ with $\Im_{(x, a)}(b, c)=C_{(x, a)}(b, c)$. We call $J$ the torsion tensor of the given linear connection on $p_{0}^{-1} E$ $\rightarrow E_{0}$.

3. Direction connections. We now define direction connections for vector bundles. Namely, a direction connection for the vector bundle 
$p: E \rightarrow M$ is a linear connection on the vector bundle $p_{0}^{-1} E \rightarrow E_{0}$ which is invariant under the diffeomorphisms of the manifold $p_{0}^{-1} E$ defined by radial expansions in the fibres of $E_{0} \rightarrow M$.

Let us describe this invariance condition precisely. Radial expansions of the fibres of $E_{0} \rightarrow M$ are diffeomorphisms of type $e \mapsto f\left(p_{0} e\right) e$, where $f$ is a smooth nonvanishing real-valued function on $M$. Tensoring such a map on the right with the identity yields a diffeomorphism of the total space of the bundle $E_{0} \otimes E \rightarrow M$. Since this total space is diffeomorphic with $p_{0}^{-1} E$, we get a diffeomorphism of $p_{0}^{-1} E$ denoted by $f$. The condition of invariance is that the connection map $D: T\left(p_{0}^{-1} E\right)$ $\rightarrow p_{0}^{-1} E$ of the given linear connection satisfies

$$
D \circ(\hat{f})_{*}=\hat{f} \circ D
$$

for all such diffeomorphisms $\hat{f}$.

Next we derive the local coordinate description of direction connections. Namely, consider a local representation $\hat{f}(x, a, b)=(x, \phi(x) a, b)$ of a diffeomorphism $\hat{f}$ defined above, where $\phi: U \rightarrow R$ is a smooth nonvanishing function. An easy calculation shows (4) to mean

$$
\begin{aligned}
\Gamma_{(x, a)}(b, \lambda)+C_{(x, a)}(b, c)= & \Gamma_{(x, \phi(x) a)}(b, \lambda)+C_{(x, \phi(x) a)}(b, \phi(x) c) \\
& +C_{(x, \phi(x) a)}\left(b, \phi^{\prime}(x)(\lambda) a\right) .
\end{aligned}
$$

Putting $c=0$ and then setting $\phi$ constant, we see that (5) is equivalent to the two conditions that

$$
\Gamma_{(x, a)} \text { is homogeneous of degree } 0 \text { in } a \text {, and }
$$

$$
C_{(x, a)}(b, c)=C_{(x, \phi(x) a)}(b, \phi(x) c)+C_{(x, \phi(x) a)}\left(b, \phi^{\prime}(x)(\lambda) a\right) .
$$

Taking $\phi$ constant, we see ( 7 ) implies

$$
C_{(x, a)} \text { is homogeneous of degree }-1 \text { in } a .
$$

Setting $\phi(x)=\exp (-f(x))$, where $f$ is a continuous linear functional on $B$ such that $f(\lambda)=1$ (Hahn-Banach Theorem), we get $C_{(x, a)}(-, a)$ $=0$, i.e.

$$
J_{e}(-, e)=0,
$$

where we identify $\left(p_{0}^{-1} E\right)_{e}$ with $E_{p e}$. As a summary, we have

Proposition 1. A linear connection on $p_{0}^{-1} E \rightarrow E_{0}$ is a direction connection iff all its local components $(\Gamma, C)$ satisfy $(6)$ and (7). Moreover, a direction connection satisfies (8) and (9).

Corollary. A linear connection on $p_{0}^{-1} E \rightarrow E_{0}$ with torsion zero is a direction connection. 
REMARK. In one of his earlier papers on Finsler geometry, $M$. Matsumoto [4] studied a more general class of linear connections on $p_{0}^{-1} E \rightarrow E_{0}$, namely, those invariant under diffeomorphisms of $p_{0}^{-1} E$ defined by uniform radial expansions in the fibres of $E_{0} \rightarrow M$, i.e. (4) holds only for constant $f$. Locally these connections are characterized by (6) and (8) (with only constant $\phi,(5)$ is equivalent to these two equations); (9) need not be satisfied. Let us call these connections weak direction connections (in [4] they were called Finsler connections). Note that they differ from ordinary direction connections only by the behavior of the torsion tensor.

4. From $D$ to $D$. Next we turn to the relationship between linear connections on $p_{0}^{-1} E \rightarrow E_{0}$ and homogeneous nonlinear connections on $p: E \rightarrow M$.

In this section we derive a necessary and sufficient condition that a linear connection $D$ on $p_{0}^{-1} E \rightarrow E_{0}$ defines a nonlinear connection $D$ on $p: E \rightarrow M$ according to the prescription in $[1, \S 5]$.

The vector bundle $p_{0}^{-1} E \rightarrow E$ has a canonical section $v: E_{0} \rightarrow p_{0}^{-1} E$ which is defined as $V(e)=e$ (using the identification $\left(p_{0}^{-1} E\right)_{e}=E_{p e}$ ). Letting $D$ denote the connection map, define the map $D: T E_{0} \rightarrow E$ as $D=r \circ D \circ V_{*}$. (This is just the covariant derivative map $Z \mapsto D_{Z} V$, see $[5, \S 2]$.)

Proposition 2. For a linear connection $D$ on $p_{0}^{-1} E \rightarrow E_{0}$, the map $D=r \circ D \circ V_{*}$ defines a nonlinear connection on $E \rightarrow M$ iff the torsion of D satisfies

$$
\mathfrak{J}_{e}(e,-)=0
$$

Moreover, if $D$ is a (weak) direction connection then the connection $D$ is homogeneous.

Proof. We work locally. $T E_{0}$ is locally $U \times E_{0} \times B \times E$ and $\mathcal{V}(x, a)=(x, a, a)$. Calculating $\mathcal{V}_{*}$ and using (3) we get $D(x, a, \lambda, b)$ $=\left(x, b+\Gamma_{(x, a)}(a, \lambda)+C_{(x, a)}(a, b)\right)$. By $[5$, Lemma 1, p. 239] this defines a nonlinear connection iff $\Gamma_{(x, a)}(a, \lambda)+C_{(x, a)}(a, b)$ is linear in $\lambda$ and independent of $b$. But this clearly occurs iff $C_{(x, a)}(a, b)=0$ for all $b$, which is precisely (10).

The local component of $D$ is thus given by

$$
\omega(x, a) \lambda=\Gamma_{(x, a)}(a, \lambda) .
$$

In case $D$ is a weak direction connection, $\Gamma_{(x, a)}$ is homogeneous of degree 0 in $a$. Since $\Gamma_{(x, a)}(b, \lambda)$ is linear in $b$, we see $\omega(x, a)$ is homogeneous of degree 1 in $a$, which means $D$ is homogeneous. Q.E.D. 
Let us say that in the setting of Proposition 2 the connection $D$ is associated to the connection $D$. This means that (11) holds for local components.

REMARK. Equation (10) is also known as E. Cartan's condition " $\mathrm{D}$ " (see [4]). This condition must be added to the results of Grifone in [3], since the step $D$ to $D$ is used there. In Dazord [1] only direction connections with symmetric torsion ("regular") are treated, so that (9) implies (10).

5. From $D$ to $D$. We now consider the reverse of the situation just discussed. Our results are as follows.

Proposition 3. For each homogeneous nonlinear connection $D$ on $E \rightarrow M$, there exists an associated direction connection $D$ with torsion zero. If $D$ is linear, the pullback $r^{-1} D$ is such $a$ D.

$D$ is not unique (even if it is assumed that dimensions are finite, $E=T M, D$ is linear and comes from a spray, and $D$ is symmetric).

Proof. Using $V E \approx p^{-1} E$ we apply the proposition of $[6,82]$, which assigns to $D$ the linear Berwald connection $D$ on $p_{0}^{-1} E \rightarrow E_{0}$. If $\omega$ denotes the local component of $D$, then that of $D$ is by definition

$$
\Gamma_{(x, a)}(b, \lambda)=\partial_{a} \omega(x, a)(b) \lambda, \quad C_{(x, a)}=0 .
$$

Hence clearly $D$ has torsion zero. If $D$ is homogeneous, then $\omega$ is homogeneous of degree 1 in $a$, whence its derivative with respect to this variable is homogeneous of one less degree, namely 0 . This means (6) holds for $\Gamma$. Since also (7) holds trivially for $C, D$ is a direction connection by Proposition 1 . Now the homogeneity of degree 1 of $\omega$ in $a$ implies by Euler's theorem that

$$
\partial_{a} \omega(x, a)(a) \lambda=\omega(x, a) \lambda .
$$

But (12) and (13) give (11), which means $D$ is associated to $D$. For $\omega$ linear in $a, \Gamma_{(x, a)}(b, \lambda)=\omega(x, b) \lambda$. This together with $C=0$ means $D$ is the pullback connection $r^{-1} D$.

To prove the nonuniqueness assertions let $M=R^{2}$ and $E \rightarrow M$ be the tangent bundle $\boldsymbol{R}^{2} \times \boldsymbol{R}^{2} \rightarrow R^{2}$ of $M$. Let $D$ be the linear connection defined by $\omega(x, a) \lambda=\langle a, \lambda\rangle x$, where $\langle$,$\rangle denotes the inner product in$ $\boldsymbol{R}^{2}$. The Berwald connection $D$ of $D$ is given by $C=0$ and $\Gamma_{(x, a)}(b, \lambda)$ $=\partial_{a} \omega(x, a)(b) \lambda=\langle b, \lambda\rangle x$. Now define a direction connection $D^{0}$ by $C^{0}=0$ and

$$
\Gamma_{(x, a)}^{0}(b, \lambda)=\left[\left(a_{1}\right)^{2} b_{1} \lambda_{1}+a_{1} a_{2}\left(b_{1} \lambda_{2}+b_{2} \lambda_{1}\right)+\left(a_{2}\right)^{2} b_{2} \lambda_{2}\right]\left(x /|a|^{2}\right),
$$

where $a=\left(a_{1}, a_{2}\right)$. Then $\Gamma_{(x, a)}^{0}(a, \lambda)=\langle a, \lambda\rangle x=\omega(x, a) \lambda$, which means $D^{0}$ is associated to $D$. But clearly $D^{\prime} \neq D^{0}$. 
Both $D$ and $D^{0}$ are symmetric, because it can be shown in a straightforward way from the definition of symmetry given in [1] that if torsion of $D$ is zero, then $D$ is symmetric iff the local component $\Gamma$ is symmetric.

To see that $D$ arises from a spray (see [1], [2]) on $M$, consider the spray defined by $G(x, a)=-\langle a, a\rangle x$. Then with $\omega$ as above, we see that $\omega(x, a) \lambda=-\frac{1}{2} \partial_{a} G(x, a)(\lambda)$, which means $D$ comes from the spray $G$. Q.E.D.

Remark. Proposition 3 shows that the uniqueness statement in Theorem 1 [2] cannot be interpreted to mean one-to-one correspondence between symmetric torsion zero direction connections and their associated sprays. Therefore, it is not clear what sense this uniqueness statement could make.

If we add the assumption that $D$ is continuously extendible to a connection on the bundle $p^{-1} E \rightarrow E$, and that $D$ is linear, then we do get uniqueness for torsion zero associated direction connections. For (as noted in [1]), the extendibility condition means locally that $\Gamma_{(x, a)}$ does not depend on $a$. Differentiation of (11) then yields $\Gamma_{(x, a)}(b, \lambda)=\omega(x, b) \lambda$, which together with $C=0$ means that $D$ is equal to the pullback connection $r^{-1} D$. (See also Matsumoto [4], where such connections are called simple connections.) However, the extendibility assumption is too restrictive for Finsler geometry.

\section{REFERENCES}

1. P. Dazord, Sur une generalisation de la notion de "spray," C. R. Acad. Sci. Paris Sér. A-B 263 (1966), A543-A546. MR 35 \#949.

2. - Connexion de direction symétrique associée a un "spray" généralisé,

C. R. Acad. Sci. Paris Sér. A-B 263 (1966), A576-A578. MR 35 \#950.

3. J. Grifone, Prolongement linéaire d'une connexion de directions, C. R. Acad. Sci. Paris Sér. A-B 269 (1969), A90-A93. MR 39 \#6228.

4. M. Matsumoto, A global foundation of Finsler geometry, Mem. Coll. Sci. Univ Kyoto Ser. A Math. 33 (1960/61), 171-208. MR 23 \#A2176.

5. J. Vilms, Connections on tangent bundles, J. Differential Geometry 1 (1967), 235-243. MR 37 \#4742.

6. Curvature of nonlinear connections, Proc. Amer. Math. Soc. 19 (1968), 1125-1129. MR 38 \#6490.

Purdue University, Lafayetre, Indiana 47907 